\title{
Cultura de la violación, un análisis del continuo en la violencia sexual que viven las mujeres
}

Culture of rape, an analysis of the continuum in sexual violence experienced by women

Sergio José Hernández Briceño

Universidad Autónoma del Estado de Morelos - México

Cuernavaca, México

sergio.hernandezb@uaem.edu.mx

\section{RESUMEN}

La violencia sexual se destaca como una de las expresiones de la Violencia Basada en Género; siendo esta expresión, una de las mayormente sufridas por las mujeres en diversas partes del mundo y en distintos ámbitos de índole familiar, laboral, social. A raíz de la presente investigación se analiza el contexto nicaragüense y mexicano, como escenarios de la región latinoamericana, donde se normalizan y justifican las diversas manifestaciones de violencias sufridas por las mujeres, todo ello a partir de una cultura de violación legitimadas por sociedades de ambos contextos. Se pretende reconocer los factores que influyen en la normalización y justificación ante situaciones de violencia sexual que sufren las mujeres, identificándose las prácticas de re victimización constantes que la sociedad proyecta ante estos escenarios de violencia hacia la mujer, afectando con ello, el desarrollo integral y autonomía de las mujeres violentadas. Es necesario reconocer las expresiones de la cultura de la violación desde una perspectiva holística y compleja que de pautas para interpretar y relacionar la misma con elementos sociales, jurídicos, cotidianos. La información recolectada aporta a determinar la forma en que el imaginario social refuerza y avala una cultura transgresora de derechos humanos; partiendo de este nivel de concientización para sustentar ideas y propuestas estratégicas que mermen los efectos de una cultura de violación. El presente artículo se elabora desde una perspectiva integral, con métodos - técnicas de investigación cualitativa y documental.

Palabras claves: Cultura de violación, Violencia sexual, imaginario social, Comportamientos, justificación.

\section{ABSTRACT}

Sexual violence stands out as one of the expressions of Gender based Violence; This expression is one of the most suffered by women in various parts of the world and in different areas of the family, work, and social nature. As a result of this research, the Nicaraguan and Mexican context is analyzed as scenarios of the Latin American region, where the various manifestations of violence suffered by women are normalized and justified, all based on a culture of rape legitimized by societies of both contexts. Among the key aspects for developing the study, it is intended to recognize the factors that influence the normalization and justification of situations of sexual violence suffered by women, identifying the constant re-victimization practices that society projects in these scenarios of violence against women, thereby affecting the integral development and autonomy of the violated women. It is necessary to recognize the expressions of the culture of rape from a holistic and complex perspective that provides guidelines for interpreting and relating it to social, legal, and everyday elements. The information collected contributes to determining the way in which the social imaginary reinforces and endorses a transgressive culture of human rights; starting from this level of awareness to support ideas and strategic proposals that reduce the effects of a culture of rape. This article is prepared from a comprehensive perspective, with qualitative and documentary research methods - techniques.

Keywords: Rape culture, sexual violence, social imaginary, behaviors, justification. 


\section{INTRODUCCIÓN}

El estudio hacia las expresiones de la cultura de violación emerge desde un análisis complejo a una de las problemáticas que siguen vigente en los escenarios sociales de la actualidad; desde la propuesta investigativa se aborda la cultura de la violación como parte de las limitantes que enfrentan las mujeres para poder acceder a la justicia ante delitos sexuales y los bloqueos sociales a los que deben enfrentarse para poder ejercer su derecho humano a vivir libres de violencia. Todo ello en el marco de elaboración de tesis de Maestría en Derecho en la Universidad Autónoma del Estado de Morelos (UAEM), periodo comprendido entre 2019 y 2020. La Violencia Basada en Género, incluye una diversidad de elementos, entornos y aspectos desventajosos para el desarrollo integral de la mujer. Por ello es imperativo analizar las manifestaciones de la violencia desde una perspectiva integral que posibilite dimensionarle como un problema de salud e interés público, para efectos de gestar soluciones viables, tangibles y proporcionables a la magnitud misma del problema.

Por lo anteriormente descrito, la presente investigación sustenta su importancia, a partir del reconocimiento hacia un entorno social y desventajoso para la mujer y su desarrollo integral. Siendo preciso frenar los estigmas, imaginarios y percepciones que normalizan y justifican tanto las expresiones de la violencia como las desigualdades de género.

Al hablar de una cultura de violación, es posible trasladarse con carácter de inmediatez hacia un abordaje meramente legislativo, sin embargo, en el contexto actual, no es posible abordar una problemática tan compleja desde una sola disciplina, para ello es necesario que se interrelacionen el que hacer antropológico, la perspectiva psicosocial y claramente el engranaje jurídico. Es precisamente en el andamiaje multi disciplinario que es prudente cuestionarse ¿Qué factores influyen en la normalización y justificación de situaciones de violencia sexual que sufren las mujeres?, ¿Cuáles son las prácticas y expresiones nocivas que implica la cultura de violación? y ¿Cómo estas últimas afectan el desarrollo integral y la autonomía de las mujeres?

La convención Belem Do Para refiere que la violencia contra la mujer constituye una violación de los derechos humanos y las libertades fundamentales, limitando total o parcialmente, el reconocimiento, goce y ejercicio de derechos y libertades de las mujeres. Lamentablemente los entornos desfavorables para las féminas han sido una constante, al punto de considerar que la sociedad tiene una deuda histórica hacia estas actoras en cuestión de reivindicación de sus derechos.

En el presente artículo se logra analizar generalidades del contexto histórico de Nicaragua y México, con respecto a la cultura de la violación y su continuo dentro de la expresión de la violencia sexual. Todas vez que se estudian las dinámicas socio comunitarias en los contextos referidos; para efectos de examinar actitudes, comportamientos, hábitos, creencias que permiten la permanencia y constancia hacia una cultura de la violación

Una vez que la comunidad lectora tome conciencia acerca de estas expresiones culturales desventajosas y reproducidas en la sociedad; será posible realizar cuestionamientos propositivos para mejorar las realidades situadas de las féminas y aminorar el impacto que conlleva la legitimación hacia una cultura de violación; la cual sustenta escenarios inequitativas y desiguales para las mujeres, indistintamente de su status, rol o condición.

\section{METODOLOGÍA}

El estudio "Cultura de la violación, un análisis del continuo en la violencia sexual que viven las mujeres". Emerge como parte de un proceso analítico y reflexivo sobre las esferas sociales y jurídicas que influye categóricamente en la reproducción de la violencia sexual desde escenarios cotidianos para las mujeres. A partir de ello es que emerge la importancia de emplear el pensamiento complejo, mismo considerado como "un paradigma relacionado a la integralidad, multidisciplinariedad, atención a las redes de apoyo y articulación de los saberes" (Copelli, 2016).

Desde este escrito serán estudiados datos estadísticos y elementos cualitativos, extraídos de diversas 
fuentes documentales; mismos que aportan a una comprensión de la problemática social referida a la cultura de la violación, sus características, las causales, repercusiones y efectos que generados en una sociedad inequitativa y desventajosa para las mujeres.

El documento refiere datos de México y Nicaragua, como escenarios latinoamericanos y a partir de los cuales, se analizan acciones, pensamientos y patrones de comportamiento machistas, que obstaculizan el acceso y ejercicio al derecho universal de las mujeres por vivir libres de violencia y en un entorno saludable y seguro. Los datos referidos para el presente estudio, se llevarán a un debido análisis desde "el método jurídico", mismo según Sánchez "viable para realizar delimitaciones y aclaraciones de los fines por los cuales quiere atenderse una problemática específica" (Sánchez, 1998, p. 97).

Para una mayor comprensión de este estudio, se precisa vincular los fundamentado en la investigativa jurídica y cualitativa, siendo esta última vista como "el intento de obtener una comprensión profunda de significados y definiciones de la situación, tal como es presentada por las personas" (Salgado, 2007, p.72). Al mismo tiempo fue empleado el método etnográfico de la ciencia antropológica, el cual

Para el desarrollo del presente artículo se hizo uso del método etnográfico, el cual consiste en "descripciones detalladas de situaciones, eventos, personas, interacciones y comportamientos que son observables" (González, 2003, p.55). Gracias a este método fue más ágil la identificación de patrones, creencias e imaginarios que persisten en los segmentos sociales y los cuales normalizan, justifican y perpetúan ciclos de violencia comunitaria. Un elemento clave para desarrollar el estudio ha sido la aplicación de la técnica de observación directa, misma definida como "aquella que consiste en la descripción sistemática de eventos, comportamientos y artefactos en el escenario social elegido para ser estudiado" (Marshall, 1989, pp.16-17). Con la técnica de observación directa ha sido posible contemplar y registrar frases, expresiones propias del imaginario social; el cual juega un rol protagónico al analizar la cultura de la violación.

En muchas de las expresiones e ideologías comunitarias, se refuerzan roles inequitativos cuyos estereotipos afianzan superioridad y sumisión de las mujeres, hacia el género masculino, todo esto desde la estructural patriarcal. Al mismo tiempo se tomaron a consideración, espacios de conversación de carácter informal, lo cual aporto a tomar en cuenta opiniones diversas por parte de mujeres y hombres hacia la problemática en estudio.

Desde la investigación documental fue realizada revisión, comprensión y reflexión sobre fuentes bibliográficas para desarrollar análisis del contenido en estudio. Sera empleado un proceso metódico de análisis - síntesis que motive la fundamentación jurídica, siendo esta comprendida como aquella cuya "naturaleza que convenza a los demás de que la tesis es indispensable llevarla a cabo. Se trata, entonces, de una justificación que interesa a la comunidad en general y no a un sujeto en particular" (Odar, 2019). A partir de esta proyección de la problemática hacia un interés general, es posible abordar distintas aristas implícitas en la cultura de violación que han sufrido las mujeres.

\section{RESULTADOS}

En el presente artículo se emplea la expresión: cultura de la violación, para referirnos a una sentida problemática social que afecta el bienestar integral de las féminas. Sin embargo, previo a analizar los aspectos epistemológicos referidos a la cultura de la violación, es preciso reflexionar y analizar de forma diferenciada las definiciones de las variables: cultura y violación. La violación según Castañeda "responde más a una necesidad de dominar y humillar a la víctima que a una supuesta estrategia reproductiva" (Castañeda, 2007, p.59). En este sentido se comprende que los detonantes para consumar el acto, radican en el fuerte deseo del agresor por dominar e intimidar a sus potenciales víctimas.

En relación a la violación como parte de la estructura de la violencia, Segato (2003) considera que el acto puede considerarse,

Un mandato de poder presente en las relaciones de género, que expresa el precepto social de que el hombre debe ser capaz de demostrar su virilidad y que, por lo tanto, el sujeto no viola porque tiene poder o para demostrar que lo tiene, sino porque debe obtenerlo (p.33). 
Sin embargo, las relaciones de poder y dominio que refieren ambos autores, no solamente encuentran un sentido para materializarse en el contacto físico, también es pertinente reconocer que la sumisión se presenta en expresiones simbólicas, donde se están perfilando el dolo, la saña y alevosía previo a consumar el acto y delito como tal. Siendo este reconocimiento importante desde el que hacer preventivo ante situaciones de violencia sexual para las mujeres.

En la violación consumada, Segato (2003), define tres referencias respecto al discurso de los violadores a la hora de comprender por qué cometen el delito; siendo estas 3 resumidas a continuación:

\begin{abstract}
1) La violación como castigo, acto disciplinador o venganza contra una mujer que abandonó su posición subordinada, desafiando con ello la posición del hombre en la jerarquía del modelo tradicional patriarcal. 2) Como agresión, desafío o afrenta contra otro hombre, usurpando parte de su patrimonio mediante la apropiación de una mujer de su posesión. 3) Como demostración de su virilidad y fuerza ante sus pares, conservando su estatus dentro de una comunidad (pp. 31-33)
\end{abstract}

Las causales afirmadas por Segato, evidencian una sentida apropiación hacia el cuerpo de las mujeres, los cuales se convierten incluso en una extensión patrimonial de los hombres quienes, en su status socialmente asignado como la figura de autoridad familiar y social, tiende a cosificar los cuerpos femeninos con quienes llegan a tener vinculo.

En este sentido, empiezan a divisarse los sesgos generados productos del sistema sexo género, trasladados en actos violatorios y en donde según Smith "un cuerpo femenino se convierte en naturalmente violable, mientras que la corpórea masculina se muestra fuerte e impenetrable” (Smith, 2004, p.168).

Desde la perspectiva jurídica, la violación ha sido definida desde su acto consumado, teniendo una conceptualización descriptiva del delito, llegando a ser considerada en la doctrina y legislaciones como "la penetración forzada de la vagina" (Muñoz, 2016, p.49). Sin embargo, los marcos legales han avanzado en diversas partes del globo terráqueo tratando de evidenciar mayor complejidad en este tipo de delito penal y eliminando la idea retrograda que implicaba en un principio, que el acto violatorio solo podría ser ejercido fuera del vínculo matrimonial.

Desde la perspectiva de los derechos humanos "la violación es considerada como un crimen de lesa humanidad" (Jerónimo, 2017, p.79), donde se involucran a un autor que invade el cuerpo de la víctima con órgano sexual (miembro viril) u otra parte del cuerpo capaz de realizar la acción o en su defecto un objeto bajo la misma finalidad. Siendo valorados para tipificar el mismo, tanto uso de la fuerza por quien comete el crimen y la falta de consentimiento por parte de la víctima.

En esta misma idea, se traen a colación las definiciones del delito de violación y violencia sexual, reflejadas en el marco legal nicaragüense y mexicano; expresando el código penal de Nicaragua, ley 641, art. 167 (2017), que realizara acto de violación quien:

Tenga acceso carnal o se haga acceder o introduzca a la víctima o la obligue a que se introduzca dedo, objeto o instrumento con fines sexuales, por vía vaginal, anal o bucal, usando fuerza, violencia, intimidación o cualquier otro medio que prive a la víctima de voluntad, razón o sentido...

En el mismo código penal se amplía el delito de violación a menores de 14 años (art. 168 CPN), mientras que en el marco de la Ley nacional 779 (2014) "Ley integral en contra de la violencia hacia la mujer", se plantea una reforma al art. 169 Código Penal de Nicaragua (2017) sobre los aspectos de la violación agravada, siendo estos:

Que el autor cometa el delito prevaliéndose de una relación de superioridad, autoridad, parentesco, dependencia o confianza con la víctima, o de compartir permanentemente el hogar familiar con 
ella; que la violación sea cometida con dos o más personas; Cuando la víctima sea especialmente vulnerable por razón de enfermedad o discapacidad física o psíquica para resistir, o se trate de una persona embarazada o mayor de sesenta y cinco años de edad; o Resulte un grave daño en la salud de la víctima. En caso de concurrir dos o más de las circunstancias descritas, se impondrá la pena máxima al actor.

De igual forma la propia ley 779, reconoce la violencia sexual como parte de las expresiones de la Violencia Basada en Género (En adelante VBG), reflejando la misma en el art. 8, inciso g y donde define que la violencia sexual es:

Toda acción que obliga a la mujer a mantener contacto sexual, físico, verbal o participar en otras interacciones sexuales mediante el uso de la fuerza, intimidación, coerción, chantaje, soborno, manipulación, amenaza o cualquier otro mecanismo que anule o limite la voluntad o su libertad sexual, independientemente que la persona agresora pueda tener con la mujer una relación conyugal de pareja, afectiva o parentesco.

Como ha podido apreciarse en el marco legal de Nicaragua, la legislación regula los delitos sexuales en dependencia del tipo de víctima, el tipo de agresor y por supuesto el modus operandis de los criminales; relacionando agravantes para interponer penas especificas cuando sean consumados los delitos contra la integridad sexual de la mujer. Sin embargo, la realidad de muchas nicaragüenses se escapa del deber ser del derecho y terminan viéndose afectadas por diversas intersecciones de violencia que dañan severamente su bienestar integral.

Un ejemplo de la exposición de la mujer nicaragüense a una cultura de violación se ha hecho notar recientemente en medio de los conflictos sociopolíticos desarrollados desde Abril 2018, donde se habla puntualmente de la escalada de la violencia simbólica, siendo expuestos los cuerpos desnudos de las mujeres en las redes sociales, existiendo como criterio para su exposición; el rol o cargo que estas féminas tuvieron en los movimientos sociales en choque (muchas de ellas fueron oficiales, funcionarias públicas, presentadoras de medios oficialistas del gobierno y demás). Este cyber delito de género y su normalización, son testimonios claves de que aún se sigue desprestigiando el cuerpo de las mujeres, considerándoles como parte de un patrimonio social y perdiéndose la perspectiva del bien jurídico a tutelar, a como es la dignidad de la mujer.

Por otra parte, en el andamiaje legal mexicano, se describe el delito de violación en el código penal federal (2020) basado en su art. 265 del siguiente modo:

\footnotetext{
Comete el delito de violación quien por medio de la violencia física o moral realice cópula con persona de cualquier sexo, se le impondrá prisión de ocho a veinte años... se entiende por cópula, la introducción del miembro viril en el cuerpo de la víctima por vía vaginal, anal u oral, independientemente de su sexo.
}

La definición en el marco legal mexicano, al igual que en el nicaragüense, se torna descriptivo del acto a sancionar, reflejándose a detalle la pena correspondiente por desarrollar este delito. De igual forma dentro de la legislación mexicana, en el marco de la ley general de acceso a una vida libre de violencia (2018), es definida la violencia sexual como:

\footnotetext{
Cualquier acto que degrada o daña el cuerpo y/o la sexualidad de la Víctima y atenta contra su libertad, dignidad e integridad física. Es una expresión de abuso de poder que implica la supremacía masculina sobre la mujer, al denigrarla y concebirla como objeto.
}

Como puede evidenciarse en ambas legislaciones (mexicana y nicaragüense), el delito de violación está considerado en la amalgama de delitos sexuales, reconociéndose en ambas marcos legales, las respectivas 
penas a definirse, una vez haya sido procesado el delito y las cuales se concretan en dependencia de los agravantes reflejados al momento de cometer el ilícito (dolo, alevosía, saña y demás). Lo cierto es que en ambas naciones se amplía la mirada hacia los delitos sexuales, precisamente para prevenir todos los posibles daños la salud física, sexual y salud reproductiva.

La violencia dentro de la pareja íntima y la violencia sexual fuera de la pareja, figuran entre las formas de violencia más generalizadas e insidiosas contra las mujeres y las niñas. El concepto de violencia hacia la mujer, incluye también las sentidas expresiones de violencia contra las niñas y adolescentes. La cultura de la violación, se enmarca en una "violencia social, misma provocadora de efectos multicausales y multiplicadores, donde se generan situaciones como el abuso sexual y la violencia doméstica” (Fragoza, 2019, p.10)

Según datos de la Organización Mundial de la Salud (OMS) al 2013 "un 35\% de las mujeres en todo el mundo han sufrido violencia física y/o sexual dentro de la pareja, o violencia sexual fuera de la pareja. Más de un $7 \%$ de las mujeres del planeta han señalado que experimentaron en algún momento violencia sexual fuera de la pareja" (OMS, 2013, p.2).

Algunos estudios promovidos por la ONU - Mujeres, refieren que hasta un "70\% de las mujeres experimentan violencia física o sexual por parte de hombres en algún momento de sus vidas, la mayoría de ellas a manos de sus esposos u otra pareja íntima" (ONU, 2013). La línea de tiempo en la cual la mujer puede ser víctima de alguna situación de violencia física y sexual, evidencia que las niñas, no están a salvo de las sentidas expresiones de la cultura de violación, de hecho, en el mismo informe de la ONU - Mujeres, se calcula que una de cada cinco niñas ha sufrido abusos durante la infancia.

Los sesgos generados a partir de las enmarcadas y desventajosas relaciones de poder, promueven modelos de crianza inseguros para las niñas, quienes son expuestas a reiterados episodios de violencia sexual. En un estudio sobre la violencia ejercida por hombres en áreas concretas de siete países de la región de Asia y del Pacifico; se puso de relieve que,

Entre un 26 y un $80 \%$ de los hombres manifestaron haber perpetrado violencia física y/o sexual contra sus parejas íntimas, y que entre un 10 y un 40\% de los hombres declararon haber violado a personas que no eran su pareja; en estos casos, la motivación citada con más frecuencia eran los derechos de apropiación sexual que ellos tenían sobre las mujeres. (ONU-mujeres, 2013, p.4).

La violencia sexual, siendo en sí misma un acto y experiencia común a la cual se enfrentan las mujeres alrededor del mundo, puede darse tanto dentro como fuera de la pareja, de ahí precisamente que sea indistinta a un espacio en específico; esto precisamente porque ya no responde a una condición domiciliar o de callejerización; la cultura de la violación y sus expresiones se tornan desde su propia naturaleza como omnipresentes.

En este sentido, es oportuno definir cuándo se está ante la violencia sexual fuera de la pareja y dentro de la relación marital. Acerca de esta última, el Estudio del secretario general de las Naciones Unidas se considera que.

\footnotetext{
La violencia sexual dentro de la pareja, comprende el contacto sexual abusivo, hacer que una mujer participe en un acto sexual no consentido y la tentativa o consumación de actos sexuales con una mujer que está enferma, incapacitada, bajo presión o bajo la influencia de alcohol u otras drogas (ONU,2006, p.111).
}

La definición brindada permite desmentir el mito o creencia de que, en los roles sexuales de la mujer dentro del vínculo matrimonial, las féminas siempre deberán estar dispuesta a responder sexualmente a los deseos de la pareja, esta idea aún tiene legitimidad en sectores rurales y urbanos donde no terminan siendo reconocidos los derechos sexuales y derechos reproductivos de las mujeres.

Al mismo tiempo es meritorio definir a que se refiere la variable de violencia sexual fuera de la pareja, 
siendo la misma considerada como aquella que es "infligida por un pariente, un amigo, un conocido, un vecino, un compañero de trabajo o un extraño" (ONU, 2006, p.10). Se incluye en la misma definición los actos que someten y obligan a otra persona en la realización de acciones sexuales, lúbricos tocamientos contra mujeres y niñas y en espacios públicos como la escuela, trabajo o la misma comunidad.

Por todo lo expresado hasta el momento, es imprescindible desde la perspectiva del pensamiento complejo y el propio enfoque holístico, reconocer que la dimensión legislativa no es indiferente a una dinámica cultural. De acá la importancia por definir también la variable cultura para el desarrollo de este artículo. Siendo considera la cultura en palabras de Tylor, citado por Harris como "ese todo complejo que comprende conocimientos, creencias, arte, moral, derecho, costumbres y cualesquiera otras capacidades y hábitos adquiridos por el hombre en tanto que miembro de la sociedad" (Harris, 2011. P.21).

La definición de cultura, permite reconocer que dentro de la amalgama de aspectos que le conforman, se gestan una serie de constructos e imaginarios sociales que tienden a legitimar la dirección, sentido e interpretación que una sociedad o comunidad, otorga a fenómenos diversos e incluso a aquellos que ante la ley versan de forma objetiva como ilícitos, terminan siendo sujetos de interpretaciones no esenciales, tal como ocurre con los actos y delitos en contra de la integridad sexual de la mujer.

Son los ya referidos constructos sociales que erotizan incluso las situaciones de violencia sexual que sufren las mujeres. En este sentido se ejemplifica la forma en que la industria pornográfica ha sexualizado las violaciones, bajo el precepto de que todas las mujeres gozan de agresiones, forcejeos e invasiones a sus espacios íntimos previo y durante el acto sexual. Reflejándose esta cultura de violación incluso cuando se presenta una eyaculación por parte del hombre en cualquier parte del cuerpo de su pareja, esto sin siquiera haber consultado y consentido el acto con antelación.

La "cultura de la violación", fue definida por primera vez durante la segunda ola del movimiento feminista, en la década del '7o. Al respecto Smith, considera que esta cultura de violación abarca

\begin{abstract}
Un conjunto complejo de creencias que alienta la agresión sexual masculina y apoya la violencia contra las mujeres. Una cultura de violación cree que la agresión sexual en los hombres está determinada biológicamente, en lugar del comportamiento aprendido. A su vez, considera que las mujeres son sexualmente pasivas y están destinadas a ser dominadas por los hombres (Smith, 2004, p.169).
\end{abstract}

La definición de Smith acerca de la cultura de la violación, reafirma el hecho de que no es posible abordar el delito de forma aislada a la cultura y sus procesos de socialización; en este sentido la dinámica cultural, que en su mayoría es machista, termina condicionando aspectos básicos de las relaciones de género como roles, comportamientos sociales, identidades asumidas y asignadas. De ahí que, desde la perspectiva social, "la cultura de la violación encuentra trasmutaciones de un contexto a otro" (CUCHE, 2002, p.6).

Según Raquel Miralles, el concepto de cultura de la violación "se ha ampliado y resignificado respecto a sus orígenes, persistiendo un discurso dominante y hegemónico que determina la forma en la que nos aproximamos a los casos de agresión sexual" (Miralles, 2020, p.83).

La cultura de la violación, según Osborne, no se remite a posiciones generalizadoras, acerca de que todos los hombres sean violadores, "sino a la legitimación de la violación en un sistema donde se trivializa esta forma de agresión, se duda de la ausencia de consentimiento, se es empático con la figura del violador y se culpabiliza a las víctimas" (Osborne, 2001, pp. 19-34). Al analizar la cultura de la violación, es posible evidenciar expresiones de normalización e indiferencia de la violencia sexual como un problema de escala proporcional. En este sentido, las diversas expresiones de la cultura de violación, terminan normando el comportamiento de las féminas, al grado que la lógica social considera que ellas son quienes deben prevenir ser abusadas por sus victimarios. Una cultura de la violación se relaciona con el constante acoso callejero e incluso se materializa como un elemento que agravia los efectos postraumáticos de las víctimas, agudizando un sentimiento de culpa, que en sí mismo no debería de existir en estas circunstancias. La vio- 
lencia sexual es una experiencia traumática, cuyas repercusiones son negativas y lesivas para el bienestar mental, físico, emocional de las mujeres afectadas por estas situaciones.

En la cultura de la violación se presume acerca de lo que pudiese haber sido el adecuado accionar de la víctima, realizándose comentarios que cuestionan el evento sin importar el estado actual físico o psicológico de la víctima. Hablar de cultura de violación se torna necesario sobre todo cuando "Una de cada cuatro mujeres y uno de cada diez hombres serán sexualmente agredidos en algún momento de sus vidas" (CCASA, 2018, p.1). El imaginario social se convierte de esta forma en un constante elemento justificador hacia los victimarios y en agravante de indefensión y re victimización para las mujeres afectadas.

Los constructos sociales dan mayor relevancia a supuestas señales emitidas por la víctima, hacia un potencial agresor violador de derechos humanos e invitan a que se desvié la atención de lo que realmente importa, a como en efecto es las afectaciones a la salud misma de la mujer. La comunidad en su desvió de atención comienzan a fijarse en aspectos irrelevantes como el tipo de vestimenta, el lugar que frecuentaba o concurría la víctima, los horarios en los que ocurrió el crimen, las personas de compañía; todo ello en afán de evidenciar los medios generadores de oportunidades que el victimario inevitablemente reconoció y tuvo que aprovechar, normalizándose así la violencia sexual a grados bochornosos.

La justificación de la VBG en cada una de sus expresiones, sobre todo en el tipo de violencia sexual, termina creando métodos ingeniosos para no evidenciar el verdadero de problema que representa esta violencia directa, indirecta, cultural y estructural, enmarcadas en el sistema patriarcal donde la sociedad se va desarrollando. En relación a la expresión de la violencia estructural, la cultura de la violación puede estar "iinstitucionalizada e interiorizada, siendo esta violencia directa también formalizada, repetitiva y ritual" (Galtung, 2018, p.168)

La cultura de la violación, afecta indistintamente a las mujeres, puesto que su motor es impulsado por la sentida cosificación al cuerpo de las féminas. Al respecto suelen emitirse comentarios despectivos para justificar las expresiones de la violencia sexual; ejemplificándose: "Debería de agradecer que la enamoren (acosen), porque agraciada no es"; "Quien la manda a tomar tanto y con puros hombres, ¿Que esperaba?”; "Ella se lo busco, por vestirse asi”; “¿Que andaba haciendo sola en ese lugar?, ipor eso le pasaron las cosas!”. Las breves frases resumidas exponen a la persona agraviada ante un claro proceso de re victimización, producto del infortunado rol de victima que protagonizo. Dejándose a un lado la transgresión directa hacia el derecho a humano a vivir libre de violencia y por supuesto el inherente derecho universal a la vida.

Ante un caso de violación u otras expresiones de la violencia sexual, el proceso de victimización no concluye con la agresión sexual, tal como refiere Garrido, esta secuencia de victimización continua "en el medio social de la víctima y tiene secuela negativa en el proceso penal, por el que necesariamente debe pasar" (Garrido, 1989, p. 108)

La cultura de la violación refiere al accionar de los potenciales agresores sexuales que suelen ser criados bajo un modelo de ejercicios de sometimiento, violencia y abuso hacia el derecho de las demás personas. Refleja el modus operandis de los agresores sexuales, a como en efecto son: drogar a sus víctimas, aislarlas y exponerlas a situaciones denigrantes y violentas al momento de cometer el ilícito. Pero este accionar no es el único ni el más frecuente para manifestar y ejercer la violencia sexual, ya que la constancia de escenarios de violencia se manifiesta en todos los ámbitos públicos y privados, expresándose como parte de las sentidas desigualdades e inequidades de género; agravándose cuando las mujeres acceden a escenarios de recreación diurnos, ya sea saliendo a ejercitarse o bien asistiendo a una fiesta nocturna con sus amistades.

En la cultura de la violación, ya no es posible referirse a escenarios específicos donde acontezcan los ilícitos, puesto que estas sentidas expresiones de violencia sexual ya no se limitan a espacios desolados, oscuros y popularmente considerados como puntos rojos, por su inseguridad. Ya que su dimensión cultura agudiza las expresiones del continuo de la violencia sexual. De ahí que sea una constante entre mujeres y para mujeres, la necesidad de notificar a sus amistades, familiares y/o parejas afectivas, el arribo a su hogar. El reconocimiento de la violencia sexual, encuentra sus sentidas vivencias y manifestaciones en un accionar ya normalizado que incluye las expresiones de acoso, tocamientos no consentidos, omisión ante 
la negativa de consumir actos sexuales (incluso tras haber afirmado previo consentimiento), miradas lascivas, invasión a espacios personales.

Todo esto debe identificarse como locuciones tangibles de la cultura de la violación; en otras palabras, los actos violatorios no solo involucran a una víctima intoxicada; no obviando por supuesto actos de sumisión química, empleado por los agresores. El imaginario colectivo en medio de sus divagaciones por no atender y atacar el verdadero problema de la violencia sexual, termina creando distinciones entre el mismo gremio de las víctimas. Expresando que a las mujeres cuyos comportamientos no son adecuados para alguien de su sexo, género y edad, terminan siendo víctimas mayoritarias de estas violencias o en otras palabras emitiendo que solo a las chicas "malas" puede pasarle esto.

Ante una cultura de violación, la decepción por un sistema ineficiente de justicia, se convierte en una constante, por ello.

\begin{abstract}
Solo una de cada tres mujeres comenta con sus familiares la experiencia; menos de dos de cada diez busca apoyo en alguna institución pública y solo el 8\% acude a las procuradurías estatales de justicia. De este porcentaje, solo tres de cada cuatro interponen una denuncia (Horizontal, 2019).
\end{abstract}

En la cultura de la violación toma especial relevancia el hecho de considerar las expresiones de acoso como actos inofensivos y merecedores de risas y bromas; sin embargo, esta normalización se traduce a expresiones relevantes como instar a que un menor desde muy temprana edad aprenda que "Robar besos" a una niña de su misma edad, es considerado un acto de hombría o bien que en el mismo sentido aprenda a "enamorar" a otras niñas. Con estos dos claros ejemplos se estimula al menor para sumarse a ser potenciales acosadores y agresores.

Al comprenderse las dimensiones y alcances de la cultura de la violación, es posible darse cuenta de que la casa y la familia, no son en realidad espacios seguros para las mujeres. Según la revista Forbes, en noviembre de 2019, el INEGI, informó que "cuatro de cada 10 mujeres (43.9\%) ha enfrentado agresiones del esposo o pareja actual o la última, a lo largo de su relación en México" (FORBES, 2020, p.1).

De igual forma el análisis hacia la cultura de la violación, se ajusta a la realidad situada, de muchas mujeres en el mundo, quienes actualmente se ven afectadas por la pandemia COVID - 19, de una forma muy distinta a las afectaciones de los hombres. De hecho, la situación actual de violencia sexual para las mujeres; se ha evidenciado exponencialmente. En México según datos de las Naciones Unidas, el número de denuncias sobre estos casos de violencia de género aumentaron.

\footnotetext{
Desde que la medida de confinamiento fue decretada, ha sido del $60 \%$, siendo de igual forma las peticiones de asilo ascendentes en un 30 \% según la Red Nacional de Refugios y en relación a los detenidos el aumento fue el $7.2 \%$ en casos de violencia intrafamiliar. Estos datos permiten desaprender la idea de que la violencia es indiferente al hogar (Romero, 2020, par. 6).
}

El imaginario social refleja a partir de la cultura de la violación, estereotipos asignados al delito de violación, el victimario y la víctima. Siendo aún más cuestionado el tipo penal y la historia de quien ha sido afectada, si la misma no se perfile o calza en algo medianamente vinculado a la perspectiva previamente construid, por ello es que el

\footnotetext{
...23\% de las mujeres violadas en México no piden ayuda en instituciones públicas por vergüenza, tres de cada cuatro por miedo, y casi una de cada cinco para evitar que la familia se entere. El silencio, el miedo y la vergüenza son los mejores aliados de los agresores. (Horizontal, 2019).
} 
El análisis de la cultura de la violación, invita a que se analicen los estímulos prácticos, ideológicos e institucionales que nutren los actos violentos hacia las víctimas y los cuales suelen ser reforzados por un complejo sistema de creencias donde se considera la misma agresión como un elementos permanente en el desarrollo de cada ser humano. Incluso desde la reflexión de la cultura de violación, es posible identificar la funesta realidad que viven muchas niñas y adolescentes a temprana edad y quienes se convierten en madres producto de una historia de violación, siendo muchas de ellas vivenciadas en sus propios hogares, por sus familiares y/o personas cercanas a la familia.

En relación a lo anterior, en Nicaragua, desde el 2018, sigue ocupando el segundo lugar entre países de Latinoamérica con la mayor tasa de mayores embarazos en adolescentes, refiriendo el dato que "por cada 1,00o mujeres que dan a luz, el 92.8 comprenden los rangos de edades entre 15 a 19 años" (LA PRENSA, 2018). La cultura de violación se manifiesta desde el momento en que estos datos suelen permanecer como problemas familiares, sin ventilarse hacia la esfera pública y así evitar episodios bochornosos para la institución familiar, todo esto a cambio de la salud, la dignidad y el bienestar de las víctimas.

El tipo de realidad que enfrenta México ante casos de violencia sexual, no dista de los escenarios nicaragüenses y de muchos países en el globo terráqueo. Puesto que, en la República Mexicana, la violencia sexual hacia la mujer en sus diversos grupos etarios, sigue permaneciendo oculta, aislada de poder ser evidenciada de las esferas públicas, en donde es meritorio que se exponga. El recelo y descontento social por parte de las víctimas, hacia las autoridades judiciales, suele ser agudizados en este tipo de casos, así lo revela un análisis realizado por la organización civil México Evalúa, según la cual,

El $99.7 \%$ de los delitos de violencia sexual contra mujeres no han sido denunciados durante la anualidad 2019. Estimándose que durante este periodo y comparando resultados con las cifras del Secretariado Ejecutivo del Sistema Nacional de Seguridad Pública (SESNSP), se estiman más de 6 millones de mujeres víctimas de algún delito sexual. (EXPANSIÓN, 2019).

Claramente la cultura de la violación, se manifiesta como un acto de discriminación hacia la mujer y donde pueden verse reflejada e interrelacionadas, aspectos que terminan agravando la propia violencia sexual e incluso dándole un sentido diverso en cuanto a la selección de las víctimas en algunos casos.

Dicho de otra forma, las violencias que sufren las mujeres estarían motivadas por diversas aristas que incluyan el estatus social, la capacidad financiera, origen étnico, religión profesada, entre otros. Esto se relaciona a lo que McCall, afirma como interseccionalidad "al término acuñado para simbolizar los matices de la discriminación de las mujeres afroamericanas, siendo aplicado para reconocer la complejidad de los procesos formales e informales que generan las desigualdades sociales" (LA BARBERA, 2016, p.36). La interseccionalidad en medio de la cultura de la violación aplica e implica la existencia de una discriminación multidireccional, para agudizar la opresión y marginación que sufren las mujeres, volviéndolas más vulnerable en su condición de víctima actual y en una futura reincidencia ante eventos que le priven de su bienestar integral. La intersección de violencia, se convierte en una amalgama de aspectos desventajosos e interrelacionados que incluye perjuicios, comportamientos y actitudes discriminatorios. En relación a estos aspectos referidos a las intersecciones de la violencia, se destaca que "la sensibilidad hacia la violencia sexual, ha sido escasa a lo largo de la historia, sobre todo cuando las víctimas eran mujeres de más baja condición sexual que sus agresores" (Koulianou, 2008, p.14).

Desde aquí el hecho de que la violencia como acto lesionador para el derecho humano a vivir la sexualidad libre, una vez vulnerado, se viva de modo diferenciado para una mujer que habita el área urbana y para una mujer que vive en comunidades rurales. Siendo para esta ultima el acceso a la justicia, probablemente más limitado por factores geográficos, étnicos y culturales.

La cultura de la violación encuentra fomento desde las estructuras sociales e industriales, las cuales generan ingresos y recursos monetarios con la promoción de esta cultura transgresora, se hace referencia a industria pornográfica, algunos videos juegos, industria musical, proxenetismo y trata de mujeres. Sin 
embargo, también se retrata en ámbitos más coloquiales y propios del folk que se incluyen dentro de la idiosincrasia local de algunos países.

En relación a las expresiones de folklore local, algunos dicharasos y refranes populares rezan: "Amarren a su gallina que mi gallo anda suelto", "Póngale una cinta roja a su hija (haciendo el símil de una can en celo)"; "La que se viste a amarillo, banano quiere"; etc. Otro accionar evidente en la cultura de la violación, se da cuando en las relaciones interpersonales, un hombre motivado por su sentir de apropiación sobre el cuerpo de las mujeres, promueve un sexting sin previo pacto, exponiendo su falo y ejerciendo con ello un claro abuso sexual ante la fémina.

En la cultura de la violación existen víctimas directas e indirectas, esto parte del hecho que la violación como un proceso de intimidación afecta tanto a las mujeres víctimas del delito, como a aquellas que nunca han sido violadas, Susan Brownmiller postula que "el miedo a la violación condiciona el comportamiento cotidiano de las mujeres” (Brownmiller, 1975, p.17).

Un dato a destacar en la cultura de la violación, es que aun cuando el tipo penal se ejerce con el gremio masculino, la forma en la que se desarrolla el crimen busca la subordinación clásica patriarcal a la que son expuestas las mujeres, Según Segato "los cuerpos masculinos, terminan siendo feminizados cuando son víctimas de este tipo de ilícito” (Segato, 2003, p.23).

Se ha hablado en este artículo acerca del imaginario social y por ello es necesario retomar sus productos más frecuentes, a como en efecto son los mitos y creencias que versan en torno a la cultura de la violación. Según Smith la "confrontación de los mecanismos que justifican la cultura de la violación y la desigualdad de derechos legales, económicos y sociales entre mujeres y hombres, pueden contribuir a eliminar el problema de la violación" (Smith, 2004, p.169).

Muchos de los mitos que versan en torno a una cultura de violación, yacen desde la propia falta de información y el silencio que versa alrededor de estas expresiones de violencia, puesto que entre menos se tiene conciencia de un problema, suele ser más fácil desvirtuarse y distanciarse de la esencia de dicha problemática, al punto de lograr hacer sentir responsables a las víctimas de estas situaciones. Según de Lonsway, "los mitos de violación son actitudes y creencias falsas que se tienen acerca de la violación persistentemente sostenida y que sirven para negar y justificar la agresión sexual del hombre contra la mujer" (Lonsway, 2008, p.58).

Por ejemplo, uno de estos referidos mitos se evidencia al momento en que un hombre no denuncia que ha sido víctima del delito de violación, precisamente por no ver cuestionada ni en vulnerabilidad su masculinidad clásica. De hecho, se considera ilusorio en algunos contextos que un hombre pueda pasar por este tipo de circunstancias. Acerca de las representaciones masculinas "la auto percepción y representación masculina, refiere que en la medida que exista una valoración cultural hacia lo hegemónico, seguirán reproduciéndose resultados que fomenten inequidad y violación de derechos” (Javier, 2004, p.24)

Un efecto negativo de los mitos sobre la cultura de la violación para las mujeres, se reflejan en aspectos que ya han venido siendo abordados a lo largo de este escrito, por ejemplo, el estilo de vestimenta, las personas que le rodean, los escenarios que frecuenta y las propias señales provocativas que la víctima envió a su agresor. Todo aquello que desvirtúa el problema en sí mismo, puede terminar siendo considerado como un mito e incluso estas mismas creencias se convierten en legitimadores de los delitos sexuales hacia las féminas, quienes no han hecho más que empezar con el proceso de victimización una vez han sido agredidas sexualmente.

Los comentarios, bromas, chistes sexistas, dichos, relatos, chismes y demás, son canales verbales y de expresión popular, por medio de los cuales mujeres y hombres inmersos en una sociedad, han promovido patrones de comportamiento y crianza que invitan a ser permisivos hacia situaciones de violencia. Sin embargo, los mismos medios y actores claves estratégicos una vez sensibilizados sobre las causas, expresiones y efectos en el marco de una cultura de violación, pueden ser gestores de cambio, cuya consigna principal sea la no justificación de las expresiones de violencia sexual u otro tipo de manifestación violenta y transgresora, bajo ninguna circunstancia. 
Desde los medios de comunicación masiva, suelen verse "legitimado y difundido discursos estigmatizadores y se ha contribuido a perpetuar los estereotipos de género, dando lugar a una especie de disciplina del terror sexual" (de las Heras, 2018, p.68)

Es necesario mermar la cultura de la violación, requiriéndose esfuerzos compartidos desde la propia perspectiva de género y el enfoque de las nuevas masculinidades, en donde los hombres dejen de normalizar situaciones de acoso y violencia en todas sus expresiones, contradiciendo la complicidad masculina, para volverse parte de la solución y no fomentadores del problema. De igual forma se requieren programas de acceso a la justicia mayormente sensibilizados en aspectos de género y que muestren empatía hacia las víctimas. Esto para hacer contrapeso a lo que en palabras Alda Facio,

Es un androcentrismo jurídico, mismo que agudiza dificultades para que las victimas asistan a una efectiva ruta de justicia, siendo revictimizadas por el personal encargado de apoyarle en su búsqueda de bienestar, contribuyendo de esta forma en la inseguridad de las víctimas. (Facio, 1999, p.11).

Dentro de las estrategias para mermar la cultura de la violación es precisa una educación basada en el consentimiento, mismo violentado en actos de ocurrencia cotidiana, por ejemplo, un niño que es obligado a saludar de beso y abrazos a alguien, siendo un mensaje directo el hecho de que no siempre podremos decidir sobre el ejercicio y apropiación de nuestros cuerpos.

El enfoque educativo para aminorar el impacto de una cultura de violación, debe aspirar a des construir lo que Kaplun refiere como "educación masculinizada, esa que tradicionalmente le damos a los niños, no es neutra ni libre; está llena de mandatos, de roles, de prohibiciones" (Medina, 2018, p.1)

La inclusión de los hombres, previamente referidas, debe estar destinada para cuestionarse los patrones de conductas violenta asumidos por ellos mismos y por sus pares en la sociedad, sus roles hegemónicos que los tienden a convertir en potenciales victimarios, a partir de conductas violentas socialmente asignadas y personalmente asumidas y un proceso judicial con garantías mínimas de sensibilización y concienciación en género.

\section{CONCLUSIONES}

El análisis de la cultura de violación, precisa estudiar de forma diferenciada, las distinciones claves: violación - cultura; esto para efectos de comprender lo complejo de esta problemática, desde una perspectiva integral en el marco de un andamiaje socio cultural. La cultura de la violación, emerge como parte de un continuo de violencia que han sufrido de forma histórica las mujeres, donde se ve involucrada una necesidad latente de dominio y transgresión hacia otros cuerpos; viéndose expuestas las mujeres a escenarios desventajosos para ejercer su derecho humano a vivir libres de violencia.

Desde la perspectiva jurídica, la violación es considerada un delito de lesa humanidad, por lo que su regulación ha sido reflejada en diversos marcos legales del globo terráqueo, como una forma de responder a la problemática. Sin embargo, es probable que se está atacando una expresión del problema y no el origen del mismo, el cual esta arraizado a la dinámica sociocultural.

Entre los factores que influyen en la normalización y justificación hacia las situaciones de violencia sexual que sufren las mujeres, destacan los de tipo cultura y su amplio bagaje de creencias, percepciones, constructos e imaginarios sociales que re victimizan a las mujeres que sufren estas sentidas expresiones de violencia, liberando al agresor de sus acciones ilícitas y delictivas.

Entre las prácticas y expresiones nocivas enmarcadas en una cultura de violación, destacan los fraseos coloquiales, dicharasos, refranes, practicas normalizadas de acoso callejero, cuestionamientos irrelevantes hacia la víctima de un delito sexual, normalización de patrones de comportamiento ofensivos y transgresores de parte de los hombres hacia la mujer, todo ello en el marco de las practicas masculinas hegemónicas. 
En el marco de la cultura de la violación, se avalan y alientan tanto creencias como prácticas que motivan la agresión sexual masculina, considerándose que las manifestaciones de violencia hacia las mujeres de parte del gremio masculino, se encuentran determinadas biológicamente, siendo motivadas por relaciones desiguales de poder entre partes dominantes (hombres) y pasivas (mujeres).

El imaginario social se convierte en un constante elemento justificador hacia los victimarios y un agravante de indefensión y re victimización para las mujeres afectadas. Expresiones populares, chistes, música, televisión y el funcionamiento de las instancias garantes de justicia, emite constantes mensajes que refuerzan en gran escala los tipos de la violencia sexual hacia la mujer. Los constructos sociales, invitan a que se desvié la atención de lo que realmente importa, a como en efecto es la salud misma de la mujer, disminuyendo la gravedad de estos delitos, disminuyendo sus efectos, precisamente para no tener que afrontar la magnitud de esta problemática y afectando con ello el bienestar integral de las féminas, sustentándose esto, ante escenarios inequitativas y desiguales para las mujeres, indistintamente de su status, rol o condición

La autonomía de las mujeres se muestra severamente afectada ante las prácticas de una cultura de violación, al grado de que el comportamiento cotidiano de las féminas puede verse condicionado por el temor a sufrir expresiones de la violencia sexual; de ahí que se considere que dichas prácticas violatorias afecten a víctimas directas e indirectas. La cultura de la violación genera consecuencias notorias a nivel mundial, en el caso del presente artículo se destaca en Nicaragua el aumento de la tasa de embarazos en adolescentes como un claro testimonio de la violencia sexual que viven las féminas y en México un excesivo porcentaje donde casi el $100 \%$ de las víctimas no denuncian sus agresiones sexuales.

Muchos de los mitos que versan en torno a una cultura de violación, yacen desde la propia falta de información y el silencio que versa alrededor de estas expresiones de violencia, entre menos se tiene conciencia de un problema, suele ser más fácil desvirtuarse y distanciarse de la esencia de dicha problemática, al punto de lograr hacer sentir responsables a las víctimas de estas situaciones.

Parte de las acciones para desvirtuar la cultura de la violación radica en procesos de sensibilización y concientización hacia la comunidad, para efectos de identificar y dimensionar la problemática misma. Es necesario la inclusión de actoras y actores estratégicos en la familia, comunidad e instituciones, que promuevan la no justificación, la prevención de la violencia sexual y su dimensión como una afectación de carácter público.

\section{REFERENCIAS}

ASAMBLEA NACIONAL DE NICARAGUA. (2014, 30 de enero). Ley no. 779, Ley integral contra la violencia hacia las mujeres y de reformas a la ley no 641 código penal con sus reformas incorporadas. Publicada en La Gaceta Diario Oficial No. 19.http://legislacion.asamblea.gob.ni/normaweb.nsf/9e314815a08d4a62062572

ASAMBLEA NACIONAL DE NICARAGUA. (2017, 5 de Julio). Ley de reforma a la ley no 641, Código penal de la república de Nicaragua ley no. 779. Publicada en La Gaceta Diario Oficial No. 126. http://legislacion.asamblea.gob.ni/normaweb.nsf/b92aaea87dac762406257265005d21f7/7373673fc384ad42062581520061e484?OpenDocument.

Brownmiller, S. (1975). Contra nuestra voluntad. Un estudio sobre la forma más brutal de agresión a la mujer: la violación. Planeta.

Castañeda, M. (2007). El machismo invisible regresa. Taurus

CCASA, ORG. (2018, 10 de Julio). Cultura de la violación. https://www.ccasa.org/wp-content/uploads/2018/o1/Cultura-de-la-Violacion.pdf.

Copelli, F. H. D. S., Oliveira, R. J. T. D., Oliveira, C. M. S. D., Meirelles, B. H. S., et al. (2016). O pensamento complexo e suas repercussões na gestão em enfermagem e saúde. Aquichan, 16(4), 501-512. http://www.scielo.org.co/pdf/ aqui/v16n4/1657-5997-aqui-16-04-00501.pdf

Cuche, D. (2002). La noción de la cultura en las ciencias sociales. Nueva Visión. http://www.proarhep.com.ar/wp-content/ uploads/Cuche_La-nocion-de-cultura-en-las-Ciencias-Sociales.pdf.

De las Heras, N. O. S. (2018). El caso de “La Manada”: cultura de la violación y Derecho penal. El Cronista del Estado Social y Democrático de Derecho, (77), 60-69. https://dialnet.unirioja.es/servlet/articulo?codigo=6667250.

Drogas \& Género. (2017, 5 de marzo). La cultura de la violación. Drogas y género https://www.drogasgenero.info/la-cultura-la-violacion/.

EI PAIS. (2019, 7 de marzo). Cultura de la violación, 4 estrategias para frenarla. EL PAIS. https://smoda.elpais.com/moda/ actualidad/estrategias-frenar-cultura-violacion/. 
Expansión Política. (2020). El $99.7 \%$ de los delitos de violencia sexual contra la mujer, no se denuncian. EXPANSIÓN POLITICA https://politica.expansion.mx/mexico/2020/01/22/el-99-7-de-los-delitos-de-violencia-sexual-contramujeres-no-se-denuncia.

Facio, A. (1999) Género y derecho, La morada, 227. http://www.bibliotecanacionaldigital.gob.cl/visor/BND:84622.

Forbes, M. (2020). Cuarentena con tu abusador: la violencia de género y el covid-19, Forbes-México. https://www.forbes. com.mx/cuarentena-con-tu-abusador-la-violencia-de-genero-y-el-covid-19/.

Fragoza, A. B. (2012). La violencia social, la violencia familiar y una mirada desde la responsabilidad social. Anuario de Psicología jurídica, 22, 127-133. https://dialnet.unirioja.es/servlet/articulo?codigo=3968730.

Galtung, J. (2016). La violencia: cultural, estructural y directa. Cuadernos de estrategia, (183), 147-168. https://Dialnet-LaViolencia-5832797.pdf.

Garrido, V. (1989). Psicología de la violación. Estudios de psicología, 10(38), 91-110. https://Dialnet-PsicologiaDeLaViolacion-66041\%20(1).pdf.

González, J. (2003). Paradigmas Emergentes Y Métodos De Investigación en el Campo de la Orientación, Opción. 27(65), 45-80.. https://www.redalyc.org/pdf/310/31021901003.pdf.

Harris, M. (2011). Antropología cultural. Alianza Editorial.

Horizontal. (2016, abril 4). La cultura de la violencia sexual en México. Horizontal.mx, https://horizontal.mx/la-cultura-dela-violencia-sexual-en-mexico-y-sus-victimas/.

Javier, P. D., \& Luisa, O. P. (2004). Género, violencia intrafamiliar e intervención pública en Colombia. Revista de estudios sociales, (17), 19-31. https://dialnet.unirioja.es/servlet/articulo?codigo=2349436.

Koulianou-Manolopoulou, P., \& Villanueva, C. F. (2008). Relatos culturales y discursos jurídicos sobre la violación. Athenea Digital. Revista de pensamiento e investigación social, (14), 1-20. https://dialnet.unirioja.es/servlet/articulo?codigo $=2736085$.

LA PRENSA. (2018, febrero 28). Nicaragua sigue ocupando la segunda tasa más alta de embarazos en adolescentes, LA PRENSA, https://www.laprensa.com.ni/2018/02/28/nacionales/2384059-nicaragua-embarazos-en-adolescentes.

Lonsway, K. A., Cortina, L. M., \& Magley, V. J. (2008). Sexual harassment mythology: Definition, conceptualization, and measurement. Sex roles, 58(9-10), 599-615. https://link.springer.com/article/10.1007\%252Fs11199-007-9367-1.

Marshall, C. (1989). Designing qualitative research. Sage. https://escholarship.org/content/qt3m25g8j8/qt3m25g8j8.pdf.

Medina, D. K. (2018). De la cultura de la violencia a la cultura de la violación. Anales del museo nacional de antropología, 20, 100-110. https://dialnet.unirioja.es/servlet/articulo?codigo $=7237267$.

Miralles, R. (2020). Cultura de la violación: una cuestión política. Libre pensamiento, (102), 82-87. https://dialnet.unirioja.es/servlet/articulo?codigo $=7407465$.

Muñoz, C. (2016). La cultura de la Violación en Chile, un análisis en los medios de comunicación digitales. [Tesis] Universidad de Chile. https://cutt.ly/PhDX2fu

Odar, R. M. T. (2019). El problema de investigación jurídica. Derecho y Cambio Social, (57), 451-503. https://cutt.ly/RhDX8GS

OMS. (2013, junio 20), informe de la OMS, destaca que la violencia contra la mujer es "un problema de salud, global de proporciones epidémicas. https://www.who.int/mediacentre/news/releases/2013/violence_against_women_20130620/ es/.

ONU. (2006). Estudio de secretario de las Naciones Unidas, poner fin a la violencia contra la mujer. NACIONES UNIDAS, Nueva York. https://www.un.org/womenwatch/daw/vaw/publications/Spanish\%20study.pdf.

Romero, M. (2020, abril 6). ONU: Violencia machista durante el confinamiento ha tenido un repunte horroroso. France 24. https://cutt.ly/XhDCwjh

Osborne, R. (2001). La violencia contra las mujeres. Realidad social y políticas públicas. UNED Ediciones. https://dialnet. unirioja.es/servlet/libro?codigo=324412.

Rios, J. (2017). Violencia sexual como crimen de lesa humanidad, casos Perú y Guatemala. Revista CIDOB d'Afers Internacionals, 117, 79-99. https://doi.org/10.24241/rcai.2017.117.3.79

Salgado, C. (2007). Investigación cualitativa: diseños y evaluación rigor metodológico y retos, Universidad San Martin de Porres. http://www.scielo.org.pe/pdf/liber/v13n13/aogv13n13.pdf.

Sánchez, R. (2012). Metodología de la Ciencia del Derecho, Ed. Porrúa.

Segato, R. (2003). Las estructuras elementales de la violencia. Universidad Nacional de Quilmes

Smith, M. (2004). Enciclopedia de la violación. Greenwood Press. 
AUTOR

Sergio José Hernández Briceño. Maestrante de Derecho en la Universidad Autónoma del Estado de Morelos (UAEM), Licenciado en Derecho en la Universidad Centroamericana (UCA), Antropólogo social de la Universidad Nacional Autónoma de Nicaragua (UNAN).

\section{Conflicto de intereses}

El autor informa de ningún conflicto de interés posible.

Financiamiento

No hay asistencia financiera de partes externas al presente artículo.

Agradecimientos

$\mathrm{N} / \mathrm{A}$ 